\title{
El régimen tributario en la formalización de las MYPES de Ica, 2016 - 2017
}

\author{
Tax regime in the formalization of MYPES \\ (micro and small businesses) of Ica, $2016-2017$
}

DOI: $10.33539 /$ consensus.2019.v24n1.2240

Ítalo Augusto Chacaliaza Uribe ${ }^{1}$

\begin{abstract}
RESUMEN
Objetivo: La investigación buscó determinar la relación existente entre el régimen tributario y la formalización de la micro y pequeña empresa en la ciudad de Ica, 2016-2017. Método: Se utilizó un cuestionario de elaboración propia con respuestas cerradas para poder recopilar la información. La población se conformó de 300 micros y pequeñas empresas de la ciudad de Ica, de la cual se obtuvo una muestra de 169 empresas que fueron sometidas a investigación bajo la técnica de muestreo aleatorio simple. Resultados: Se encontró correlación positiva significativa $(r=0,621)$ entre la inscripción ante la SUNAT y el pago de los tributos, además, correlación positiva significativa $(r=0,062)$ entre la presentación de las declaraciones juradas y la formalización de trabajadores; así mismo se evidenció correlación positiva muy significativa $(r=0,337)$ entre la presentación de las declaraciones juradas y el llevado de los libros contables. Finalmente se encontró correlación positiva muy significativa $(r=0,736)$ entre la formalización de las empresas y el aumento de clientes, así mismo se evidenció que existe correlación positiva $(r=0,818)$ con el fortalecimiento de las relaciones con los proveedores. Conclusión: Se concluye que existe una relación positiva muy significativa $(r=0,638)$ entre el régimen tributario y la formalización de las micro y pequeña empresa en la ciudad de Ica, 2016- 2017.
\end{abstract}

\section{Palabras clave}

Tributos, régimen tributario, formalización, empresa, MYPE

\begin{abstract}
Objective: The research sought to determine the relation between the tax regime and the formalization of Mypes in the city of Ica, 2016- 2017. Method: A questionnaire, with closed-ended answers, was used to collect the information. The population of interest was consisted of 300 Mypes in the city of Ica, from which a sample of 169 businesses was obtained and subjected to research, using the simple random sampling technique. Results: A significant positive correlation $(r=0.621)$ between the registration before SUNAT (National Superintendency of Tax Administration) and the payment of the taxes was found, as well as, a significant positive correlation $(r=0.062)$ between the presentation of the affidavits and the formalization of workers; Likewise, a very significant positive correlation ( $r=0.337$ ) was found between the presentation of the affidavits and the registry of the accounting books. Finally, a very significant positive correlation $(r=0.736)$ was found between the formalization of the businesses and the increase of clients, likewise it was evidenced that there is a positive correlation ( $r=0.818)$ with the strengthening of relations with suppliers. Conclusion: It is concluded that there is a highly significant positive relationship $(r=0.638)$ between the tax regime and the formalization of Mypes in the city of Ica, 2016- 2017.
\end{abstract}

\section{Keywords}

Tributes, tax regime, formalization, business, MYPE

Contador Público por la Universidad Nacional San Luis Gonzaga de Ica, egresado de la Maestría en Política y gestión Tributaria con mención en Política y Sistema Tributario por la Universidad Nacional Mayor de San Marcos. Lima, Perú. 


\section{INTRODUCCIÓN}

En la ciudad de Ica, la mayoría de las empresas que se encuentran en funcionamiento pertenecen a la categoría de las micro y pequeñas empresas (MYPES) debido a la poca cantidad en el volumen de sus ventas y actividades que realizan. Dichas empresas en su mayoría se limitan solo a ejercer el comercio dentro de la provincia de Ica, teniendo entre sus principales rubros empresas dedicadas a servicios de limpieza, mantenimiento y comercio. Cabe resaltar, que también existen empresas que se encuentran registradas en Ica, pero realizan sus labores en otro lugar, estas especialmente se dedican a la minería.

Asimismo, el nivel de conocimiento acerca del régimen tributario es mínimo, la gran cantidad de empresarios desconocen las normativas vigentes acerca de lo concerniente a políticas tributarias y cultura tributaria. Esta desinformación conlleva a que los empresarios no opten por la formalización, debido a un concepto primitivo, según el cual, el pagar impuestos perjudicaría a su economía; sin embargo, no toman conciencia que, al contribuir con el país mediante los impuestos se realiza una inversión para las obras que se desarrollen en beneficio de ellos mismos.

El estado peruano planteó un régimen tributario que estuvo vigente hasta el año 2016; en el 2017 optó por realizar modificaciones en beneficio de los empresarios para que puedan formalizarse, permitiéndoles mejores beneficios y tasas diferentes, las cuales se adecuen a sus ingresos y egresos. Aún a pesar de los esfuerzos realizados por el estado existe la informalidad de algunas empresas y otras que, si bien están inscritas en los entes encargados, realizan evasión de impuestos por desconocimiento o por beneficio personal, afectando de esta manera el correcto desarrollo de la formalización de las empresas.

Sin embargo, debido a las nuevas modificatorias se ha podido observar un incremento en la cantidad de empresas que han formalizado. Por lo tanto, esta investigación busca encontrar la relación entre el régimen tributario y como este influye positivamente en la formalización de las micro y pequeñas empresas (MYPES).
La empresa se crea con la finalidad de iniciar un negocio o actividad productiva o de servicio para obtener beneficios económicos, el cual se puede iniciar de manera personal, como una persona natural o recurrir a alguna de las fórmulas societarias para mediante una persona jurídica realizar las actividades. Schumpeter (1978) considera que la empresa es la generación de nuevas combinaciones, y empresario es el individuo que se encarga de dicha generación. El empresario es la persona con iniciativa y que muestra capacidad para proponer y realizar combinaciones novedosas para producir, por lo que, la empresa es un proceso radical de innovación en sí mismo.

Dentro del análisis económico clásico la empresa es el ente de la producción encargado de la transformación de materiales, esfuerzo, energía y demás inputs en productos y/o servicios, los cuales han de ser distribuidos entre consumidores. La empresa clásica es, consecuentemente, la que se encarga de producir el proceso de transformación de inputs en outputs de acuerdo con lo señalado por González y Ventura (2003).

Montoya (2004) indica que la empresa es una universalidad, es una agrupación de derechos o cosas las cuales se mantienen independientes, y en su conjunto conforman una unidad especial a la cual se le puede brindar tratamiento jurídico unitario. Las MYPES abarcan gran parte de las empresas que realizan algún tipo de actividad económica en el país, son de vital importancia ya que aportan gran cantidad del PBI, y también debemos considerar que en muchas ocasiones este tipo de empresas aportan al autoempleo ya que una persona es la que posiciona su negocio o actividad y empieza obtener ingresos.

Silupú (2011) considera que, en el caso del Perú, las micro y pequeñas empresas se conforman por unidades económicas de carácter legal que pueden ser conformadas por personas naturales o jurídicas las cuales cumplen con sus obligaciones fiscales, por lo que se encuentran inscritas en el RUC y eligen alguno de los regímenes vigentes para el cumplimiento de sus obligaciones tributarias por sus rentas de tercera categoría, entre los que encontramos personas naturales y jurídicas que realicen actividad comercial. Toyama (2008) indica que, del total de empresas 
en Perú, cerca del $98 \%$ son microempresas la cuales representan el $42 \%$ del PBI y emplean al $55 \%$ de los trabajadores. Solo el $9 \%$ goza de beneficios laborales y acceso a atención en salud. Las pequeñas empresas, en nuestro país, emplean al $27 \%$ de los trabajadores y de dicho grupo solo el $25 \%$ tiene beneficios laborales.

Según el Decreto legislativo $N^{\circ} 1086$, se establecen características que deben cumplir las empresas para ser consideradas micro y pequeña empresa. Caroy (2011) indica que la normatividad de las MYPES clasifica a dicho sector considerando dos características: nivel de ingresos y el número de trabajadores. Sin embargo, dichas características han variado desde septiembre de 2008 con la promulgación de la Ley MYPE actual.

El problema general de la investigación fue: ¿Cómo influye el régimen tributario en la formalización de la micro y pequeña empresa en la ciudad de Ica, 2016- 2017?

Asimismo, los problemas secundarios fueron:

¿De qué manera la inscripción en la SUNAT de la micro y pequeña empresa incrementa el pago de tributos en la ciudad de Ica, 2016-2017?

¿En qué medida la presentación de declaraciones juradas influye en la formalización de trabajadores y el llevado de registros contables de la micro y pequeña empresa en la ciudad de Ica, 20162017 ?

¿De qué manera la formalización de la micro y pequeña empresa mejoran las relaciones comerciales con otras empresas de la ciudad de Ica, 2016- 2017?

Con relación al objetivo general se planteó, determinar como el régimen tributario influye en la formalización de la micro y pequeña empresa en la ciudad de Ica, 2016- 2017.

Asimismo, los objetivos específicos fueron:

Establecer de qué manera la inscripción en la SUNAT de la micro y pequeña empresa incrementan el pago de tributos en la ciudad de Ica, 2016- 2017.
Identificar en qué medida la presentación de declaraciones juradas influye en la formalización de trabajadores y el llevado de registros contables de la micro y pequeña empresa en la ciudad de Ica, 2016- 2017.

Determinar de qué manera la formalización de la micro y pequeña empresa mejoran las relaciones comerciales con otras empresas de la ciudad de Ica, 2016- 2017.

\section{MATERIAL Y MÉTODOS}

La investigación es cuantitativa, debido a que utilizó la recolección de datos para probar hipótesis con base en la medición numérica y el análisis estadístico, con el fin establecer pautas de comportamiento y probar teorías. (Hernández, Fernández y Baptista, 2014). La presente investigación reúne las condiciones para ser considerada como investigación básica o teórica. Asimismo, de acuerdo con el propósito del estudio se centra en una investigación de nivel descriptivo y correlacional.

Las empresas motivo de estudio fueron micro y pequeñas empresas que realizan su labor en la ciudad de Ica y que están inscritas en la SUNAT, así como aquellas que no han sido aún registradas por dicha institución, pero, sin embargo, se tiene conocimiento que realizan actividades comerciales. La población estuvo constituida por 300 micros y pequeñas empresas de la Ciudad de Ica. Para determinar el tamaño de la muestra se usó la técnica de muestreo aleatorio simple utilizando la siguiente fórmula:

$$
\mathrm{n}=\frac{\mathrm{Z}^{2} \mathrm{~N} p q}{\mathrm{E}^{2}(\mathrm{~N}-1)+\mathrm{Z}^{2} \mathrm{pq}}
$$

En Donde: $\mathrm{n}=$ Tamaño de la muestra

$\mathrm{N}=$ Población $(300)$

$Z=$ Nivel de confianza (1.96)

$\mathrm{p}=$ Tasa de prevalencia de objeto de estudio (0.50)

$\mathrm{q}=(1-\mathrm{p})=0.50$

$\mathrm{E}=$ Error de precisión 0.05 
Por lo tanto:

$$
\begin{array}{cc}
\mathrm{n}= & \frac{(1.96)^{2}(300)(0.50)(0.50)}{(0.05)^{2}(300-1)+(1.96)^{2}(0.50)(0.50)} \\
\mathrm{n}= & \frac{288.12}{0.7475+0.9604} \\
\mathrm{n}= & \frac{288}{1.7079} \\
\mathrm{n}= & 169
\end{array}
$$

Para la recolección de datos se aplicó una encuesta de elaboración propia, que permitió obtener la información necesaria para medir las variables planteadas. Asimismo, las encuestas fueron realizadas en el lugar de ejercicio de la empresa, al encargado que se encontró en ese momento o en algunos casos al titular de la empresa. Se complementó con entrevistas a expertos relacionados al tema, quienes nos dieron su punto de vista respecto al tema en análisis, y nos brindaron su opinión profesional respecto del panorama actual de las MYPES y su formalización.

La información obtenida se procesó utilizando el Programa Estadístico SPSS para realizar el tratamiento estadístico de los resultados. Asimismo, se realizó la comprobación de hipótesis y una vez obtenidos los resultados se realizaron gráficos y tablas para una mejor representación, finalmente se concluyó con los resultados que se desprenden de la presente investigación.

La hipótesis general planteada fue:

H1: Existe relación positiva significativa entre el régimen tributario y la formalización de la micro y pequeña empresa en la ciudad de Ica, 20162017.

H0: No existe relación positiva significativa entre el régimen tributario y la formalización de la micro y pequeña empresa en la ciudad de Ica, 2016- 2017.

Así, como las hipótesis específicas fueron:

H2: Existe relación positiva significativa entre la inscripción de las micro y pequeña empresa ante la SUNAT y la recaudación por tributos en la ciudad de Ica, 2016- 2017.
H3: Existe relación positiva significativa entre la presentación de declaraciones juradas y la formalización de los trabajadores, así como con el llevado de registros contables de la micro y pequeña empresa en la ciudad de Ica, 20162017.

H4: Existe relación positiva significativa entre la formalización de las micro y pequeña empresa y la mejora en la realización de sus operaciones comerciales con otras empresas en la ciudad de Ica, 2016 - 2017.

\section{RESULTADOS}

A continuación (Tabla 1) se muestran los resultados obtenidos de la aplicación de la encuesta a la muestra seleccionada.

Tabla 1

Resultado de las encuestas

\begin{tabular}{lcccc}
\hline \multirow{1}{*}{ Pregunta } & \multicolumn{4}{c}{ Respuesta } \\
\cline { 2 - 5 } & SI & $\%$ & NO & $\%$ \\
\hline Inscripción en la SUNAT & 140 & $82.84 \%$ & 29 & $17.16 \%$ \\
Régimen Tributario & 140 & $82.84 \%$ & 29 & $17.16 \%$ \\
Pago de Tributos & 110 & $65.09 \%$ & 59 & $34.91 \%$ \\
$\begin{array}{l}\text { Presentación de } \\
\text { declaraciones juradas }\end{array}$ & 117 & $69.23 \%$ & 52 & $30.77 \%$ \\
$\begin{array}{l}\text { Formalización de } \\
\text { Trabajadores }\end{array}$ & 154 & $91.12 \%$ & 15 & $8.88 \%$ \\
$\begin{array}{l}\text { Llevado de libros } \\
\text { contables }\end{array}$ & 75 & $48.08 \%$ & 81 & $51.92 \%$ \\
$\begin{array}{l}\text { Relaciones comerciales } \\
\text { Aumento de Clientes }\end{array}$ & 112 & $66.27 \%$ & 57 & $33.73 \%$ \\
$\begin{array}{l}\text { Fortalecimiento Relación } \\
\text { con proveedores }\end{array}$ & 96 & $53.25 \%$ & 79 & $46.75 \%$ \\
\hline
\end{tabular}

De los resultados obtenidos frente a la pregunta sobre si la empresa se encuentra inscrita en la SUNAT con su Registro Único de Contribuyente (RUC), se obtuvo que 140 empresas si se encuentran inscritas, lo cual representa el $82.84 \%$ de la muestra seleccionada, por otro lado, 29 empresas de la muestra no se encuentran inscritas en la SUNAT, representando el $17.16 \%$. 
Por lo cual se desprende que la mayoría de las empresas evaluadas sí se encuentran inscritas en la SUNAT.

De la pregunta sobre si la empresa se encuentra acogida a alguno de los regímenes tributarios, tales como el Nuevo RUS, Régimen especial, Régimen MYPE Tributario o el Régimen General, se obtuvo que 140 empresas si se encuentran acogidas a alguno de los regímenes mencionados, lo cual representa el $82.84 \%$ de la muestra seleccionada, por otro lado, 29 empresas de la muestra no se encuentran acogidas a alguno de los diferentes regímenes existentes, lo cual representa el $17.16 \%$.

Los resultados obtenidos frente a la pregunta sobre el pago de tributos de parte de la empresa, se obtuvo que 110 empresas si realizan el pago correspondiente de sus tributos, lo cual representa el $65.09 \%$ de la muestra seleccionada, por otro lado, 59 empresas de la muestra no realizan el pago de sus tributos, representando el $34.91 \%$.

En referencia a la presentación de las declaraciones juradas a la SUNAT, se obtuvo como resultado que 117 empresas si realizan sus declaraciones juradas correspondientes, lo cual representa el $69.23 \%$ de la muestra seleccionada, por otro lado, 52 empresas de la muestra no realizan la presentación de manera oportuna de las declaraciones juradas correspondientes a la SUNAT, representando el $30.77 \%$. De la evaluación de resultados se observa que gran parte de la muestra sí realizan la presentación de manera oportuna de las declaraciones juradas ante la SUNAT.

De la pregunta sobre si la empresa que cuenta con trabajadores, estos han sido declarados oportunamente ante la SUNAT, se obtuvo que 154 empresas sí han declarado a sus trabajadores de manera oportuna, lo cual representa el $91.12 \%$ de la muestra seleccionada, por otro lado, 15 empresas de la muestra no han realizado la declaración de sus trabajadores ante la SUNAT, lo cual representa el 8.88\%. El resultado a la pregunta sobre si la empresa realiza el llevado de libros contables correspondientes según su régimen, se obtuvo que 75 empresas sí realizan el adecuado llevado de libros contables correspondientes, lo cual representa el $48.08 \%$ de la muestra seleccionada, por otro lado, 81 empresas de la muestra no realizan el llevado de libros contables que se encuentra obligado a llevar, lo cual representa el $51.92 \%$.

Se obtuvo como resultado con relación a si la formalización de su empresa le ha permitido mejorar sus relaciones comerciales, que 112 empresas sí ha mejorado sus relaciones comerciales, a diferencia de 57 empresas de la muestra que no han mejorado sus relaciones comerciales luego de la formalización de su empresa. La mayoría de las empresas evaluadas han mejorado sus relaciones comerciales luego de la formalización.

En relación a las preguntas referentes a si la empresa ha incrementado su cantidad de clientes luego de formalizarse y si la empresa ha fortalecido sus relaciones comerciales con sus proveedores, se observa que para el caso de la primera pregunta se obtuvo que 90 empresas tuvieron incremento de clientes, frente a 79 empresas que indican lo contrario; referente a la segunda pregunta 96 empresas indican que han fortalecido sus relaciones comerciales, sin embargo, 73 empresas indican no se obtuvo dicho fortalecimiento.

Se encontró correlación positiva muy significativa $(\mathrm{r}=0.621)$ entre la inscripción a la SUNAT y la realización del pago de tributos, ver Tabla 2. Lo cual significa que, a mayor inscripción en la SUNAT, se realiza un mayor pago en lo concerniente a tributos.

Tabla 2

Correlación entre la inscripción a la SUNAT y el pago de tributos

\begin{tabular}{cccc}
\hline & & $\begin{array}{c}\text { Inscripción } \\
\text { SUNAT }\end{array}$ & $\begin{array}{c}\text { Pago de } \\
\text { tributos }\end{array}$ \\
\hline $\begin{array}{c}\text { Inscripción } \\
\text { SUNAT }\end{array}$ & $\mathrm{r}$ & 1 &, $621^{* *}$ \\
& $\mathrm{p}$ & &, 000 \\
Pago de tributos & $\mathrm{r}$ &, $621^{* *}$ & 1 \\
& $\mathrm{p}$ &, 000 &
\end{tabular}

Luego de analizar los resultados se evidenció correlación positiva significativa $(r=0.062)$ entre 
la presentación de las declaraciones juradas y la formalización de los trabajadores, lo cual significa que, a mayor presentación de las declaraciones juradas, se realiza una mayor formalización de los trabajadores, ver Tabla 3.

Tabla 3

Correlación entre la presentación de las declaraciones juradas y la formalización de los trabajadores

\begin{tabular}{cccc}
\hline & & $\begin{array}{c}\text { Declaraciones } \\
\text { Juradas }\end{array}$ & $\begin{array}{c}\text { Formalización } \\
\text { de trabajadores }\end{array}$ \\
\hline $\begin{array}{c}\text { Declaraciones } \\
\text { Juradas }\end{array}$ & $\mathrm{r}$ & 1 &, 062 \\
& $\mathrm{p}$ & &, 420 \\
$\begin{array}{c}\text { Formalización de } \\
\text { trabajadores }\end{array}$ & $\mathrm{r}$ &, 062 & 1 \\
& $\mathrm{p}$ &, 420 & \\
\hline
\end{tabular}

En la Tabla 4 de acuerdo con el análisis de los datos se halló correlación positiva muy significativa $(r=0.337)$ entre la presentación de las declaraciones juradas y el llevado de los libros contables correspondientes a cada régimen, lo cual significa que, a mayor presentación de las declaraciones juradas, se realiza una mayor incidencia en el llevado de los libros contables correspondientes.

Tabla 4

Correlación entre la presentación de las declaraciones juradas y el llevado de libros contables

\begin{tabular}{cccc}
\hline & & $\begin{array}{c}\text { Declaraciones } \\
\text { Juradas }\end{array}$ & $\begin{array}{c}\text { Libros } \\
\text { contables }\end{array}$ \\
\hline $\begin{array}{c}\text { Declaraciones } \\
\text { Juradas }\end{array}$ & $\mathrm{r}$ & 1 &, $337 * *$ \\
$\begin{array}{c}\text { Libros } \\
\text { contables }\end{array}$ & $\mathrm{p}$ &, $337 * *$ & 1 \\
& $\mathrm{p}$ &, 000 & \\
\hline
\end{tabular}

Se encontró que existe una correlación positiva muy significativa $(r=0.736)$ entre la formalización de la empresa y el aumento de clientes, lo cual significa que, a mayor prevalencia de formalización de la empresa, se realiza un incremento en el número de clientes. Tal como se puede apreciar en la Tabla 5.
Tabla 5

Correlación entre la formalización de las empresas y el aumento de clientes

\begin{tabular}{cccc}
\hline & & $\begin{array}{r}\text { Formalización } \\
\text { de la empresa }\end{array}$ & $\begin{array}{c}\text { Aumento de } \\
\text { clientes }\end{array}$ \\
\hline $\begin{array}{c}\text { Formalización de } \\
\text { la empresa }\end{array}$ & $\mathrm{r}$ & 1 &, $736^{* *}$ \\
& $\mathrm{p}$ & &, 000 \\
$\begin{array}{c}\text { Aumento de } \\
\text { clientes }\end{array}$ & $\mathrm{r}$ &, $736^{* *}$ & 1 \\
& $\mathrm{p}$ &, 000 & \\
\hline
\end{tabular}

De la información procesada se evidenció una correlación positiva muy significativa $(r=0.818)$, entre la formalización de la empresa y el fortalecimiento de relaciones con los proveedores, lo cual significa que, a mayor prevalencia de formalización de la empresa, se realiza un mayor fortalecimiento de las relaciones comerciales con los proveedores (Tabla 6).

Tabla 6

Correlación entre la formalización de las empresas y el fortalecimiento de relaciones con los proveedores

\begin{tabular}{cccc}
\hline & & $\begin{array}{c}\text { Formalización } \\
\text { de la empresa }\end{array}$ & $\begin{array}{c}\text { Relación con } \\
\text { proveedores }\end{array}$ \\
\hline $\begin{array}{c}\text { Formalización de } \\
\text { la empresa }\end{array}$ & $\mathrm{r}$ & 1 &, $818^{* *}$ \\
& $\mathrm{p}$ & &, 000 \\
$\begin{array}{c}\text { Relación con } \\
\text { proveedores }\end{array}$ & $\mathrm{r}$ &, $818^{* *}$ & 1 \\
& $\mathrm{p}$ &, 000 & \\
\hline
\end{tabular}

De acuerdo con el análisis de los resultados del presente estudio se halló correlación positiva muy significativa $(r=0.638)$, entre el régimen tributario y la formalización de la empresa, lo cual significa que a mayor prevalencia de empresas que se acogen al régimen tributario, existe una mayor formalización de las empresas (ver Tabla 7). 
Tabla 7

Correlación entre el régimen tributario y la formalización de las empresas

\begin{tabular}{cccc}
\hline & & $\begin{array}{c}\text { Régimen } \\
\text { tributario }\end{array}$ & $\begin{array}{c}\text { Formalización } \\
\text { de la empresa }\end{array}$ \\
\hline $\begin{array}{c}\text { Régimen } \\
\text { tributario }\end{array}$ & $\mathrm{r}$ & 1 &, $638^{* *}$ \\
& $\mathrm{p}$ & &, 000 \\
$\begin{array}{c}\text { Formalización } \\
\text { de la empresa }\end{array}$ & $\mathrm{r}$ &, $638^{* *}$ & 1 \\
& $\mathrm{p}$ &, 000 & \\
\hline
\end{tabular}

\section{DISCUSIÓN}

En la presente investigación, se encontró que existe una relación positiva muy significativa entre el régimen tributario y la formalización de las micro y pequeñas empresas, a diferencia de lo propuesto por Salinas (2010) que hace referencia a que el estado genera una enorme presión tributaria; sin embargo, en la investigación no se incluyeron empresas del sector minero. Cabe resaltar que, en consonancia con Sandoval, Posso y Quispe (2012) las MYPES cumplen un rol significativo en el desarrollo económico del país.

Además, existe una relación positiva muy significativa entre la inscripción ante la SUNAT y el pago de los tributos de las micros y pequeñas empresas, en contraste con lo encontrado por Tarazona y Veliz (2016) ya que la diferencia entre el nivel de cultura tributaria entre la ciudad de Ica y otras ciudades es mayor, lo cual permite un aumento de formalización.

Adicionalmente, la SUNAT se encarga de brindar facilidades para que las micros $y$ pequeñas empresas puedan formalizarse. Asimismo, el pago puntual de los tributos según el cronograma permite evitar multas a la empresa en consonancia con lo propuesto por Romero (2016).

En relación con lo antes mencionado también se encontró que existe una relación positiva significativa entre la presentación de las declaraciones juradas y la formalización de trabajadores; así como una relación positiva muy significativa entre la presentación de las declaraciones juradas y el llevado de los libros contables correspondientes, lo cual fortalece lo propuesto por Romero (2016) acerca de la seriedad y confiabilidad que dan las empresas luego de formalizarse.

Finalmente, se observa una relación positiva muy significativa entre la formalización de las empresas y el aumento de clientes de las micro y pequeñas empresas, debido a que las perciben como empresa serias $y$ responsables según lo propuesto por Romero (2016), así como una relación positiva muy significativa entre la formalización de las empresas y el fortalecimiento de las relaciones con los proveedores, lo cual, según el autor aludido, también contribuye a tener mayores posibilidades de obtener préstamos que le permitan invertir en su empresa.

\section{CONCLUSIONES}

Luego del análisis de los datos obtenidos, se llega a las siguientes conclusiones:

1. Existe relación positiva muy significativa $(r=0,638)$ entre el régimen tributario y la formalización de las micro y pequeñas empresas en la ciudad de Ica, 2016- 2017.

2. Existe relación positiva muy significativa $(r=0,621)$ entre la inscripción ante la SUNAT y el pago de los tributos de las micro y pequeñas empresas de la ciudad de Ica, 2016- 2017.

3. Existe relación positiva significativa $(\mathrm{r}=0,062)$ entre la presentación de las declaraciones juradas y la formalización de trabajadores de las micro y pequeñas empresas de la ciudad de Ica, 2016- 2017.

4. Existe relación positiva muy significativa $(r=0,337)$ entre la presentación de las declaraciones juradas y el llevado de los libros contables correspondientes de las micro y pequeñas empresas de la ciudad de Ica, 2016- 2017. 
5. Existerelación positiva muysignificativa $(\mathrm{r}=0,736)$ entre la formalización de las empresas y el aumento de clientes de las micro y pequeñas empresas de la ciudad de Ica, 2016- 2017.
6. Existe relación positiva muy significativa $(\mathrm{r}=0,818)$ entre la formalización de las empresas y el fortalecimiento de las relaciones con los proveedores de las micro y pequeñas empresas de la ciudad de Ica, 2016- 2017. 


\section{REFERENCIAS}

Caroy, M. (2011). Redefiniendo la política de promoción de las MYPE en las compras estatales. Lima, Perú: Derecho PUCP, 66, 287-299.

Gonzáles, E. y Ventura, J. (2003). Fundamentos de administración de empresas. Madrid, España: Ediciones Pirámide.

Hernández, R., Fernández, C. y Baptista, P. (2014). Metodología de la investigación. México: Mc Graw Hill.

Montoya, U. (2004). Derecho Comercial (Tomo I). Lima, Perú: Editorial Jurídica Grijley.

Romero, G. (2016). Incidencia de los pagos de los tributos para la obtención de beneficios en las MYPES comerciales del parque industrial del distrito Villa El Salvador (Tesis de pregrado). Universidad Autónoma del Perú, Lima.

Salinas, E. (2010). Efectos tributarios en la gestión de las MYPES de la actividad minera del oro en la Región de Arequipa (Tesis de maestría). Universidad de San Martín de Porres, Lima, Perú.

Sandoval, K., Posso, K. y Quispe, C. (2012). El régimen legal peruano de las micro y pequeñas empresas y su impacto en el desarrollo nacional (Tesis de maestría). Universidad Peruana de Ciencias Aplicadas, Lima, Perú.

Schumpeter, J. (1978). Teoría del desenvolvimiento económico. México D.F.: Fondo de Cultura Económica.

Silupú, B. (2011). Fortalecer para crecer, casos de éxito del programa MYPE. Piura, Perú: Universidad de Piura.

Tarazona, I. y Veliz, S. (2016). Cultura tributaria en la formalización de las MYPES, Provincia de Pomabamba- Ancash año 2013 (Caso: micro empresa T\&L S.A.C.) (Tesis de pregrado). Universidad de Ciencias y Humanidades, Lima. Perú.

Toyama, J., Irujo, C. (2008). La nueva reforma de las microempresas. Comentarios al régimen laboral establecido por el D.S. Nº07- 2008- TR (Tomo 181). Lima, Perú: Actualidad Jurídica.

Fecha de recepción: 06-06-19.

Fecha de aceptación: 30-06-19. 